\title{
Práctica de medicina tradicional en la comunidad afrodescendientes creole de Bilwi
}

\author{
Erna Patterson Rankin
}

La medicina tradicional de la etnia creole sustenta la salud en el equilibrio cálido/frío y el pensamiento animista del mundo. Su metodología parte de explicar el sentido holístico de la vida. Los agentes tradicionales de salud asumen su rol como un don divino y basan su intervención en la restitución del equilibrio con rituales y plantas medicinales.

Los resultados obtenidos en esta investigación han sido de gran satisfacción ya que hemos podido obtener la información de las medicinas tradicionales; elaborar un mapeo de quiénes la practican, y deteminar a qué área de la medicina tradicional se dedican.

\section{Médicos tradicionales afrodescendientes de Bilwi}

Estos especialistas son reconocidos como: bush healers, bone fixer, midwife, sipritual guide. Son nombres que tienen desde antaño. Esto lo dejó claro uno de los entrevistados que dijo recordar esos calificativos desde la época en que vivían sus abuelos y abuelas, lo que significa que también estos nombres son también parte de la historia.

Los creoles de Bilwi mantienen arraigado aún el uso de plantas medicinales en los barrios en que tienen mayoría, tales como Arlen Siu, Spanistown, El Muelle, Los ángeles. De éstos, los que más practican estos conocimientos ancestrales son los creoles del barrio Spanistown. Se descubrió que solo el $20 \%$ de los entrevistados con conocimientos de medicina tradicional ejerce esta práctica de forma franca y directa para la atención al pueblo, o sea, que atienden en sus casas y cobran por su trabajo. El resto, lo aplica en el núcleo familiar y a nivel de sus amistades. Sin embargo es importante subrayar que la mayoría dijo no sentirse curanderos, más bien, conocedores y practicantes de una medicina casera que es parte de la medicina tradicional.

\section{Plantas medicinales que más utilizan los médicos tradicionales creoles}

Las plantas medicinales más utilizadas por la comunidad afro de Bilwi son: basil (albahaca), valerian (valeriana), sorosi, fever grass o lemon grass (zacate de limón), (ginger) gengible, sepol tree (eucalipto o sepol), thime (tomillo), guava (guayaba), almond (almendra), spanish ella, mery gold, (flor de muerto o san diego), john charles (escobilla o escoba amarga), vervaine (verbena), jackaass biters, coco muma, rangoat dashalam, pis a bed, (pico de pájaro), chrismas bloosom (ceroncontil), margan (orégano orejón, orégano castilla), sour orange tree (naranja agria).

De este listado que mencionaron son nueve especies las que utilizan con mayor frecuencia: destacando lemon grass (zacate de limón), basil (albahaca), chrismas bloosom (ceroncontil), margan (orégano orejón, orégano castilla), jackas bittters, sorosi, eucalipto.

\section{Enfermedades que más afectan a la población creole}

Fiebre, catarro, tos, diarrea, tuberculosis y reumatismo, esto lo mencionaron las personas mas adultas, presión alta y, también, baja, diábetes, anemia, artritis, asma o cansancio, stroks, diarrea, piojos 


\section{Utilización de plantas medicinales según enfermedades}

\section{ALBAHACA / BASIL}

Propiedades curativas: se utiliza para eliminar parásitos intestinales, fiebre, dolor de oído, granos, dolor de cabeza y para baños espirituales.

Partes utilizables: flores y hojas.

Modo de preparación: té, baños.

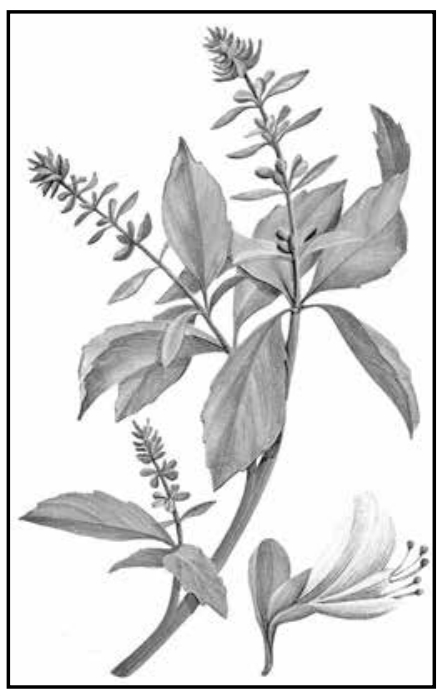

\section{CRISTMAS BLOOSOM}

Propiedades curativas: se utiliza como purgante o laxante, cuando hay constipación, escabiosis, y manchas blancas de la piel (liver spots), este término se usa para señalar que el hígado está sucio.

\section{Partes utilizable: hojas.}

Modo de preparación: té, baño y pasta.

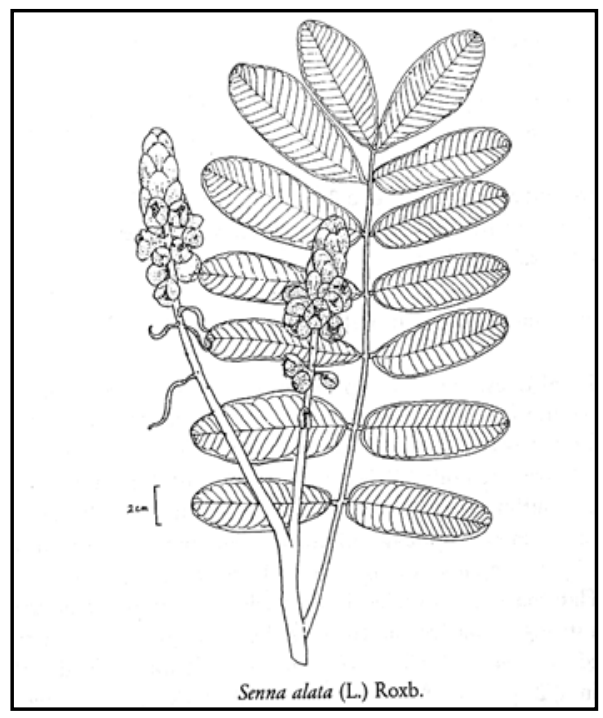

\section{EUCALIPTO / EUCALIPTUS}

Propiedades curativas: se utiliza como descongestionante nasal y para la tos.

Partes utilizables: hojas.

Modo de preparación: té, infusión.

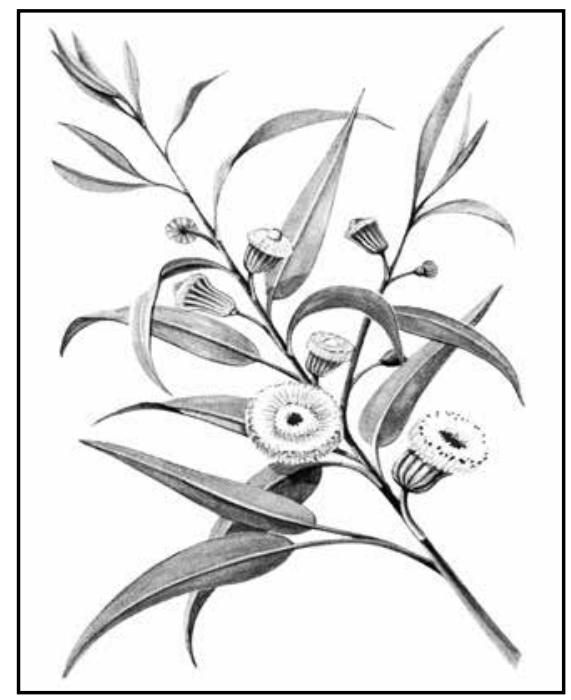

\section{JOHN CHARLES (hierba martin)}

Propiedades curativas: para gripe, fiebre y tos, parásitos intestinales; también, para lavar los granos y lavados vaginales.

Partes utilizables: hojas.

Modo de preparación: té.

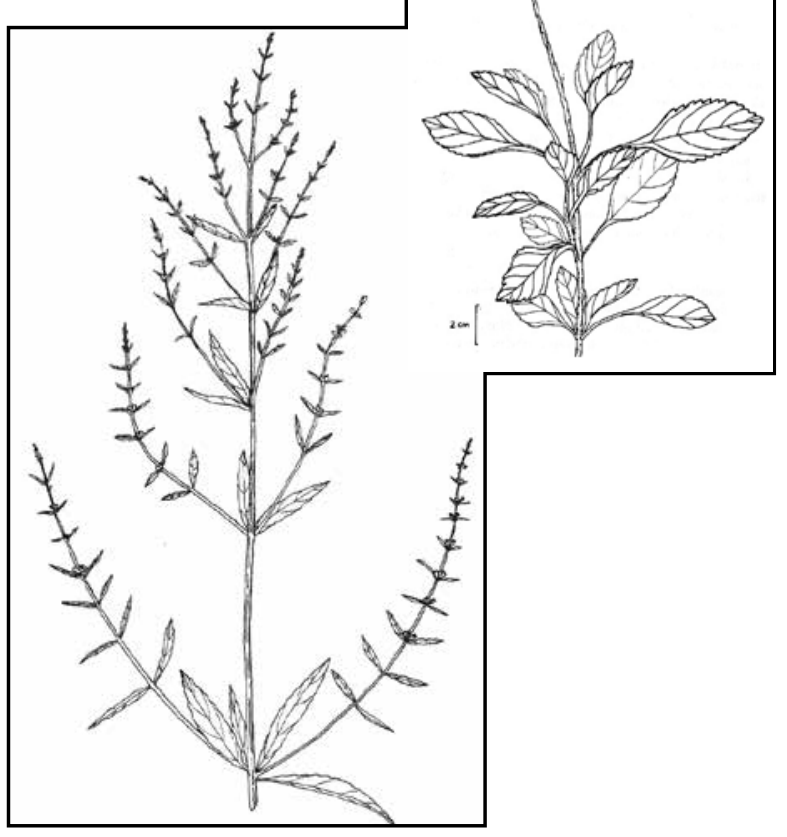




\section{THYME (TOMILLO)}

Propiedades curativas: ayuda a disminuir la inflamación de las amígdalas, para esto se debe hacer gárgaras. Ayuda en la digestión, sirve para la tos, y es un fabuloso ingrediente para la comida.

Partes utilizables: hojas.

Modo de preparación: té y cocimiento.

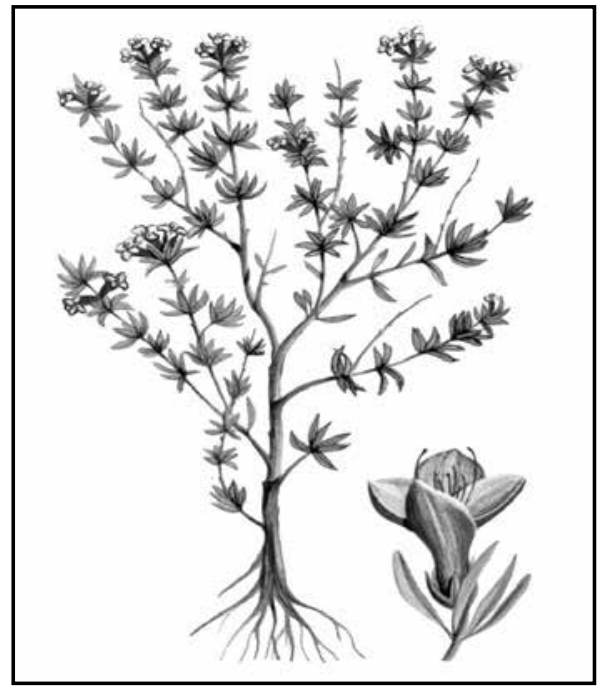

\section{SOROSI}

Propiedades curativas: purifica y disminuye el azúcar en la sangre de los diabéticos, también sirve para lavar granos de la piel y de la boca.

Partes utilizable: hojas.

Modo de preparación: Té, cocimiento.

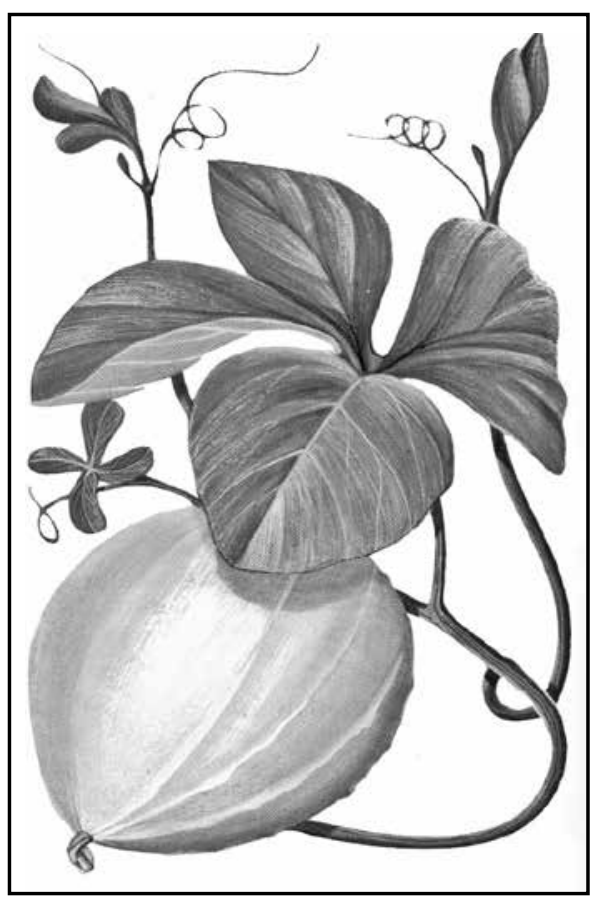

\section{ZACATE DE LIMÓN.}

Propiedades curativas: para fiebre y catarro, falta de respiración. Se recomienda endulzar con miel de abejas.

Partes utilizables: hoja y rizoma.

Modo de preparación: cocimiento.

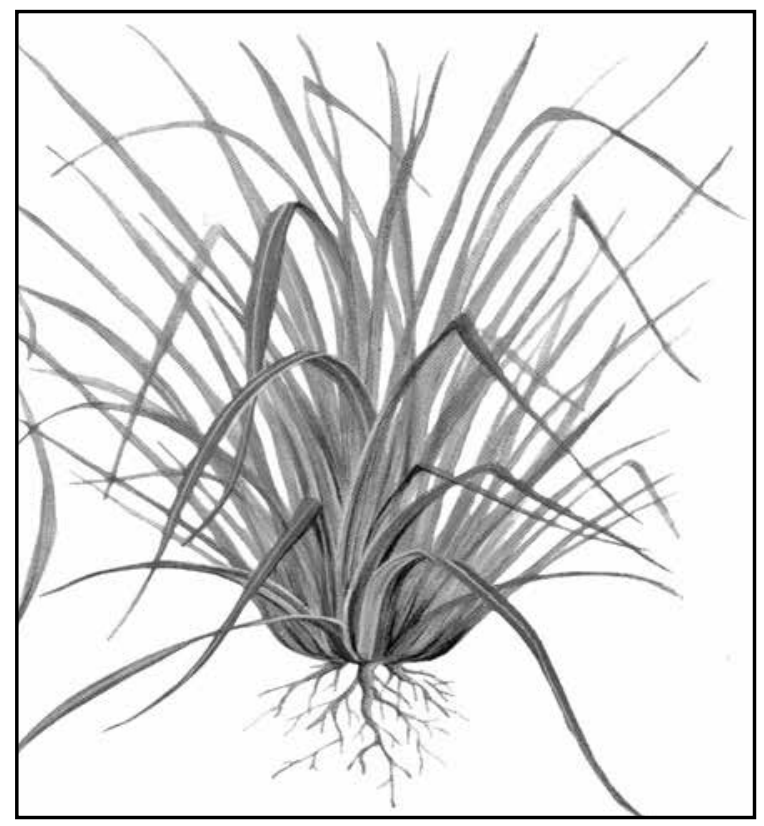

\section{JACKAS BITTTERS}

Propiedades curativas: para infección en la piel, granos, piojos y escabiosis.

Partes utilizables: hojas

Modo de preparación: cocimiento, cataplasma.

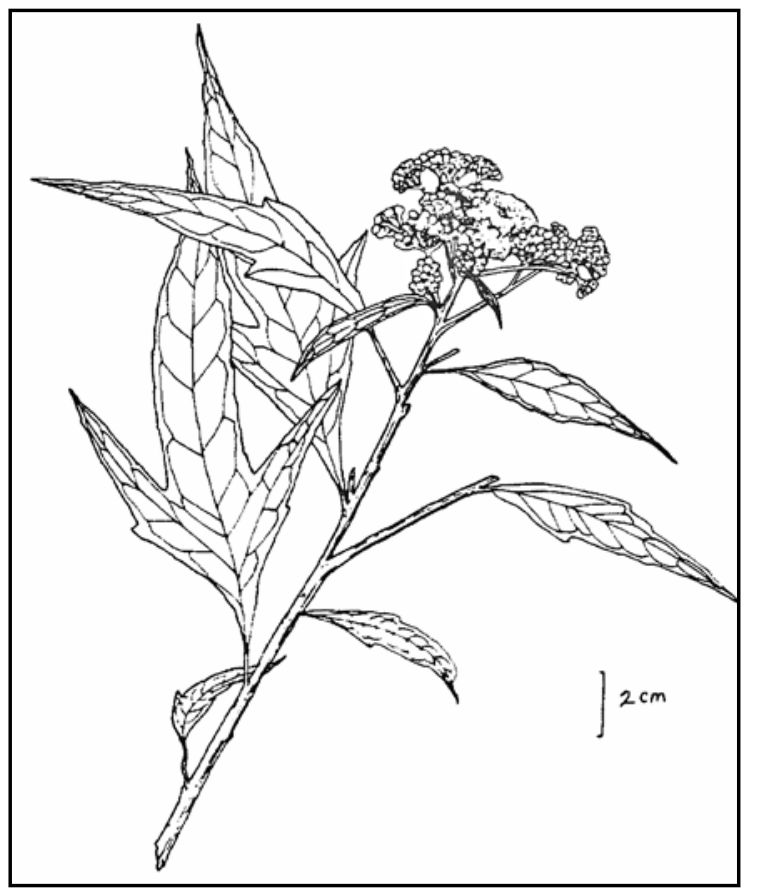




\section{Procedimiento para la preparación de la plantas}

Existen diferentes formas de preparar las plantas. Éstas pueden ser en forma de té, cocimiento, y en pasta o cataplasma, así como baños e inhalación. Todos los entrevistados mencionaron que las plantas tienen efectos para la sanación de las personas enfermas.

La mayoría dijo hacer uso de plantas medicinales ya que esto lo hacían sus padres cuando ellos eran pequeños. Le daban té de diferentes tipos, incluso, este trato que recibían por parte sus padres influyó mucho y así es como hoy ellos continúan utilizando esta alternativa con sus hijos y nietos; también sus esposas fueron atendidas por parteras.

Algunos dijeron tener sus pequeños huertos de plantas medicinales en sus predios, pero éstos eran la minoría, sin embargo el resto dijo que tienen en sus patios algunas especies. Se debe de señalar que los creoles son más conservadores en las prácticas debido a que esto se reduce a su círculo familiar ya que la mayoría no cobra por este servicio.

\section{Referencias Bibliográficas}

Arvigo, R. \& Balick, M, J. (1998). Rainforest remedies: One hundred healing herbs of Belize. (2a ed.).USA: Lotus Press.

Arvigo, R. \& Epstein, N. (2001). Rainforest home remedies: The Maya Way to heal your body and replenish your soul. (2a. ed.). San Francisco: HarperCollins

Barret, B. (1994). Salud y cultura en el Caribe nicaragüense: Interacciones etnomédicas. Wani, (15), 20-38.

Centro Ecuménico Antonio Valdivieso. (s.f.). Mujer y salud: Rescate de la medicina tradicional de San Francisco Libre. Managua: CEAV.

Cunningham Kain, M. \& Cunnigham, W. (1994). Medicina tradicional en comunidades miskitas de Waspam, Río Coco, $R A A N$. Managua: CEM.

Guatemala. Asociación de Servicios Comunitarios de Salud. (1988). Plantas medicinales en la salud de la comunidad. Chimaltenango, Guatemala:ASECSA.

House, P. (1995). Plantas medicinales comunes de Honduras. Tegucigalpa: UNAH.

Nicaragua. Asamblea Nacional. Decreto No. 3584-2003: Reglamento a la ley No. 28. (2003. 2 de octubre). La Gaceta, (186). pp. 4910-4921.

Nicaragua. Asamblea Nacional. Ley No. 759, Ley de Medicina Tradicional Ancestral. (2011, 04 de julio). La Gaceta, (123). pp. 4028-4034.

Nicaragua. Centro de Educación Promocional Agraria.(1984). La salud al alcance del pueblo con medicina natural: Microdosis. Managua: CEPA

Sosa Gómez, R. (1998). El poder medicinal de las plantas. Madrid: API.

Universidad de las Regiones Autónomas de la Costa Caribe de Nicaragua, Instituto de Medicina y Desarrollo Comunitario. (s.f.). Manual de plantas medicinales para el tratamiento de enfermedades comunes.Puerto Cabezas, Nicaragua: URACCAN. 